\title{
High prevalence of coexisting prehypertension and prediabetes among healthy adults in northern and northeastern China
}

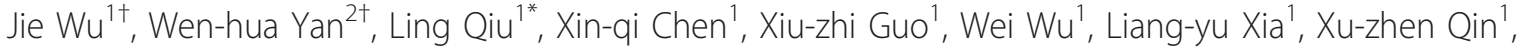
Yan-hong Liü ${ }^{3}$, Hai-tao Ding ${ }^{4}$, Shao-mei Han ${ }^{5}$, Cheng-li Xu ${ }^{5}$ and Guang-jin Zhu ${ }^{5}$

\begin{abstract}
Background: Prehypertension and prediabetes are major risk factors of cardiovascular disease, and their combined presence may result in more serious cardiovascular outcomes than expected with either prehypertension or prediabetes alone. The aim of the present study was to evaluate the prevalence of coexisting prehypertension and prediabetes, and the associated risk profiles in a Chinese population.

Methods: A cross-sectional survey in a representative sample of 3,595 men and 4,593 women aged 18 years and older was performed between 2008 and 2010. Prehypertension and prediabetes were diagnosed using the guidelines from the Seventh Report of the Joint National Committee on prevention, detection, and treatment of high blood pressure and American Diabetes Association, respectively. Prehypertension was defined as a systolic blood pressure of 120-139 $\mathrm{mmHg}$ and/or diastolic blood pressure of $80-89 \mathrm{mmHg}$, and prediabetes was defined as a fasting blood glucose of $5.6-6.9 \mathrm{mmol} / \mathrm{L}$.

Results: The prevalence of coexisting prehypertension and prediabetes was $11.0 \%$. Men had a higher prevalence of coexisting prehypertension and prediabetes than women (14.2\% vs. 8.4\%; $P<0.0001)$. This prevalence increased with age and body mass index, and was the lowest among Mongolian-Chinese (5.1\%). A multivariate analysis showed that $\gamma$-glutamyltransferase and uric acid were significantly and positively correlated with body mass index, waist circumference, blood pressure, triglycerides, and total cholesterol, and negatively correlated with high density lipoprotein cholesterol in subjects with prehypertension and prediabetes.

Conclusions: There is a large proportion of Chinese adults with coexisting prehypertension and prediabetes. Thus, there is a need for more efforts that implement public health programs that target the earlier stages of hypertension and diabetes.
\end{abstract}

\section{Background}

Cardiovascular disease (CVD) is considered to be a major cause of death in most developed and developing countries [1]. Hypertension is a major risk factor of CVD. Even a slightly elevated blood pressure within the normal range is associated with cardiovascular morbidity and mortality [2]. Therefore, in 2003, the Seventh Report of the Joint National Committee on the

\footnotetext{
* Correspondence: lingqiubj@yahoo.com.cn

+ Contributed equally

'Department of Clinical Laboratory, Peking Union Medical College Hospital, Peking Union Medical College \& Chinese Academy of Medical Science, Beijing 100730, China

Full list of author information is available at the end of the article
}

Prevention, Detection, Evaluation, and Treatment of High Blood Pressure (JNC-7) introduced a new category: prehypertension (PreHTN), where systolic blood pressure (SBP) is from 120 to $139 \mathrm{mmHg}$, and/or diastolic blood pressure (DBP) is from 80 to $89 \mathrm{mmHg}$ [3]. Individuals with PreHTN are more susceptible to developing true hypertension and coronary atherosclerosis [4]. Several national blood pressure surveys in the United States and other countries report that more than $30 \%$ of the general adult population has PreHTN [5,6]. Prediabetes (PreDM) is another important risk factor of CVD, which is associated with impaired fasting glucose (IFG) and/or glucose tolerance (IGT). According to the American 
Diabetes Association, PreDM is indicated by IFG, where serum glucose concentrations range from $5.6 \mathrm{mmol} / \mathrm{L}$ $(100 \mathrm{mg} / \mathrm{dl})$ to $6.9 \mathrm{mmol} / \mathrm{L}(125 \mathrm{mg} / \mathrm{dl})$, as well as IGT, where serum glucose concentrations range from 7.8 $\mathrm{mmol} / \mathrm{L}(140 \mathrm{mg} / \mathrm{dl})$ to $11.1 \mathrm{mmol} / \mathrm{L}(199 \mathrm{mg} / \mathrm{dl}) 2 \mathrm{~h}$ after a $75 \mathrm{~g}$ oral glucose load [7]. IFG is associated with insulin resistance and an increased risk of cardiovascular pathology. Although less risky than IGT, IFG is coupled with a greater conversion from PreDM to overt diabetes (i.e. approximately $24 \%$ in less than three years) [8]. Recently published data on a large population-based sample of more than 45,000 people, aged 20 years and older, in China indicated that the prevalence of PreDM was $15.5 \%$ [9].

Given that the effects of certain clinical precursors, such as PreHTN and PreDM, on the cardiovascular system are often distinct, their combined presence in the same patient may result in more severe coronary artery disease than expected with either PreHTN or PreDM alone [10]. The probable CVD risk with PreHTN and PreDM, to some extent, is dependent on whether PreHTN leads to hypertension and PreDM leads to diabetes. Additionally, racial and ethnic differences are known to influence the prevalence and risks of PreHTN and PreDM [11,12]. Several large surveys have yielded prevalence estimates for either PreHTN or PreDM in China [9,13]. Nevertheless, there is little information available in the literature regarding the prevalence of coexisting PreHTN and PreDM (co-PreHTN and PreDM) among individuals located in areas inhabited by Chinese ethnic minorities. Furthermore, to the best of our knowledge, there have been no population-based, cross-sectional studies that have assessed the epidemiology of co-PreHTN and PreDM among healthy Chinese adults. Thus, the aim of the present study was to evaluate the prevalence of co-PreHTN and PreDM, along with their associated risks, in populations located in northern and northeastern China.

\section{Methods}

\section{Study population}

A population-based, cross-sectional survey of the Chinese Physiological Constant and Health Condition (CPCHC) was conducted between 2008 and 2010. Representative samples of the general Chinese population, aged 18 years and older, from the Hei Longjiang Province and Inner Mongolian Autonomous Region in mainland China were determined according to a random, multistage cluster sampling scheme, allowing for good prevalence estimates of the Chinese population. Two urban and two rural areas were selected from each province. Written informed consent was obtained from each participant prior to data collection. The protocol was approved by the Institutional Review Board of the Institute of Basic Medical Sciences, Chinese Academy of Medical Sciences. Trained medical personnel collected information on risk factors via questionnaires (e.g. demographic, socioeconomic, and healthrelated information), and obtaining anthropometric measurements and blood samples for biochemical assessments.

\section{Inclusions and exclusions}

The 2008-2010 CPCHC samples included 29,639 apparently healthy participants. Those who suffered from systemic disease involving diabetes mellitus, hypertension or other cardiovascular, renal, gastro-intestinal, pulmonary disease or cancer were excluded. Moreover, participants taking any medication known to affect carbohydrate and lipid metabolism were also excluded. A schematic of the screening process is presented in Figure 1. Of the total number of participants, $44.3 \%(n=13,140)$ were selected randomly to complete blood testing. Of 8,475 adults, aged $\geq 18$ years, 287 (3.4\%) had missing data on blood pressure (BP) and/or laboratory tests. Therefore, the final sample size of disease-free healthy adults was 8,188 (3,595 men and 4,593 women), of which 5,788 were Han Chinese, 936 were Korean-Chinese, 1,237 were Mongolian-Chinese, and 227 were of other ethnicity. Their age distribution was as follows: 3,214 were aged 18-39 years, 1,762 were aged 40-49 years, 1,580 were aged 50-59 years, and 1,632 were aged > 60 years.

\section{Data collection and anthropometry}

Epidemiological data were collected on all subjects via a standard questionnaire, which included demographic characteristics (i.e. age, gender, and ethnicity), socioeconomic data (i.e. educational level, marital status, and occupation), past history, and lifestyle risk factors. Smoking status was classified as non-smokers, current smokers (i.e. daily smoking regardless of the amount and type), and ex-smokers. Alcohol drinking status was defined as non-drinkers, current drinkers (frequent consumption of alcohol regardless of the amount and type), and ex-drinkers.

Body weight was measured to the nearest $0.1 \mathrm{~kg}$ on a calibrated beam scale and height was measured barefoot in triplicate using a wall-mounted stadiometer to the nearest $0.1 \mathrm{~cm}$. Body mass index (BMI; an index of overall obesity) was calculated as body weight (in kilograms) divided by height (in meters squared). BMI was categorized according to the World Health Organization criteria, where a BMI of $<25 \mathrm{~kg} / \mathrm{m}^{2}$ is considered normal, a BMI between 25 and $29 \mathrm{~kg} / \mathrm{m}^{2}$ is considered overweight, and a $\mathrm{BMI} \geq 30 \mathrm{~kg} / \mathrm{m}^{2}$ is considered obese [14]. Waist circumference (WC; a surrogate marker for central adiposity) was measured midway between the lower rib margin and the iliac crest at the end of a gentle expiration.

$\mathrm{BP}$ was measured following a resting period of at least 10 min using an electronic sphygmomanometer (OMRON, HEM-7000). The participant's arm was placed 


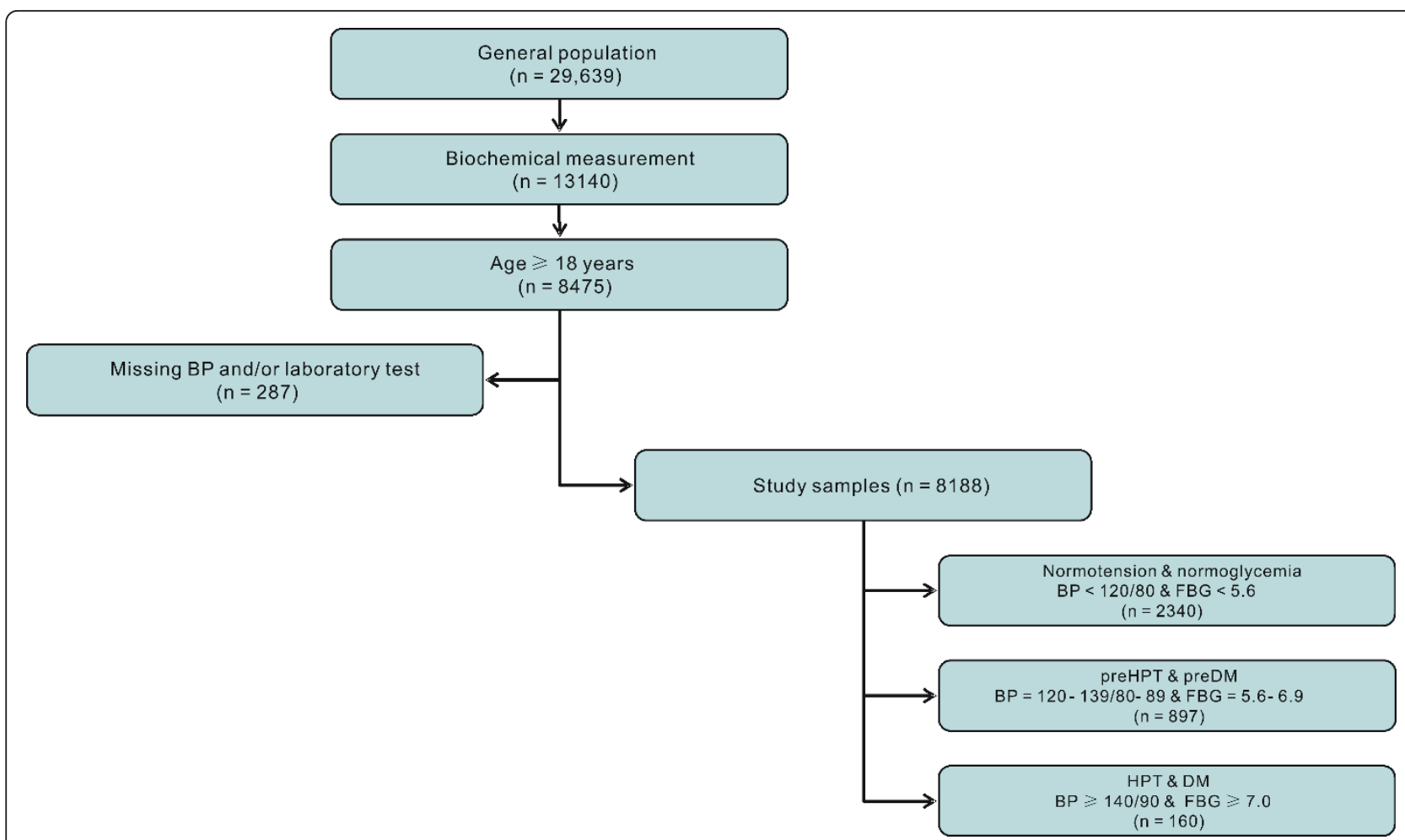

Figure 1 A schematic used for screening and inclusion of the study sample. A total of 29,639 disease-free and healthy individuals were recruited between 2008 and 2010, and 13,140 individuals had biochemistry measurements collected. Of the 8,475 adults aged $\geq 18$ years, 287 participants had missing data on BP and/or laboratory tests, and as result were excluded. The final sample size was 8,188, which consisted of 2,340 individuals with both normotension and normoglycemia, 897 individuals with coexisting prehypertension and prediabetes, and 160 individuals with both hypertension and diabetes. Abbreviations: BP, blood pressure; FBG, fasting blood glucose; PreHTN, prehypertension; PreDM, prediabetes; HTN, hypertension; DM, diabetes mellitus.

at the level of the heart, and BP was measured three times. The averages of the three measurements were used. If a subject was hypertensive, then a review was performed by doctors to exclude secondary hypertension.

\section{Laboratory measurements}

All procedures were performed following a 12-h overnight fast. Blood was drawn from the antecubital vein of the right arm. Serum $\gamma$-glutamyltransferase (GGT; a sensitive marker of alcohol intake and hepatic inflammation) and uric acid (UA; a marker of inflammation and metabolic syndrome), both of which play important roles in the development of cardiovascular events, were assayed with an Olympus AU2700 Automatic Biochemical Analyzer and Olympus agent (Olympus, Tokyo, Japan). Fasting blood glucose and lipid profiles, including total cholesterol (TC), triglycerides (TG), high-density lipoprotein cholesterol (HDL-C), and low-density lipoprotein cholesterol (LDL-C) were also assessed. The biochemical laboratories participating in the survey followed the same internal quality control program that was standardized by the Peking Union Medical College Hospital.
Diagnosis and classification of diabetes and hypertension

Diagnosis of PreHTN was based on the criteria in the JNC-7 report [3]. Specifically, PreHTN was defined as a SBP of 120-139 $\mathrm{mmHg}$ and/or a DBP of $80-89 \mathrm{mmHg}$, whereas normotension was defined as a SBP $<120 \mathrm{mmHg}$ and a DBP $<80 \mathrm{mmHg}$, and hypertension was defined as a $\mathrm{SBP} \geq 140 \mathrm{mmHg}$ and/or a DBP $\geq 90 \mathrm{mmHg}$.

Diagnosis of PreDM was based on the criteria of the American Diabetes Association [7]. Pre-DM was defined as fasting blood glucose (FBG) levels from $100 \mathrm{mg} / \mathrm{dl}(5.6$ $\mathrm{mmol} / \mathrm{L})$ to $125 \mathrm{mg} / \mathrm{dl}(6.9 \mathrm{mmol} / \mathrm{L})$ ], which indicated IFG, and/or 2-h postprandial blood glucose (PBG) levels from $140 \mathrm{mg} / \mathrm{dl}(7.8 \mathrm{mmol} / \mathrm{L})$ to $199 \mathrm{mg} / \mathrm{dl}(11.1 \mathrm{mmol} / \mathrm{L})$ following a $75 \mathrm{~g}$ oral glucose load, which indicated IGT. Individuals with $\mathrm{FBG}<5.6 \mathrm{mmol} / \mathrm{L}$ were considered normal, whereas individuals with FBG $\geq 7.0$ or 2 -h PBG $\geq 11.1$ were diagnosed with diabetes. Fasting was defined as no caloric intake for at least $8 \mathrm{~h}$.

\section{Statistical analysis}

Data were entered and documented on EpiData 3.1 software (The EpiData Association, Odense, Denmark). Datasets were transferred into an SPSS compatible format. 
Data are presented as counts and percentages \pm standard errors (SE) for categorical variables, and means \pm standard deviation (SD) for continuous variables with a normal distribution. Comparisons between groups were made using an analysis of covariance. Medians and interquartile ranges of GGT and TG were calculated due to their abnormal distributions, and comparisons between these groups were made using the Wilcoxon rank sum test. Prevalence (\%) indicates the percentage of healthy Chinese men and women with a condition at the time of data collection, and means indicate the average value of a characteristic in healthy Chinese adults. Correlation analyses were performed using either the Pearson or Spearman correlations. Statistical analyses were performed on the statistical software, SPSS version 13.0 (SPSS Inc., Chicago, Illinois, USA). All tests for statistical significance were two-tailed, and considered significant when $P<0.05$.

\section{Results}

\section{Epidemiology}

Of the 8,188 disease-free adults, 897 (11.0 \pm 0.3\%) had coPreHTN and PreDM, 160 (2.0 $\pm 0.2 \%)$ had concurrent hypertension and diabetes mellitus, 2,340 (28.6 $\pm 0.5 \%)$ had both normotension and normoglycemia, and the remaining 4,791 had other combinations of resting BP and fasting serum glucose concentrations as shown in Table 1.

The prevalence of co-PreHTN and PreDM was higher in the Hei Longjiang province compared to the Inner Mongolian Autonomous Region (13.3\% vs. 8.8\%, $P<$ 0.0001 ), as shown in Table 2. Men had a higher prevalence of co-PreHTN and PreDM than women (14.2\% vs. $8.4 \%, P<0.0001)$. The prevalence increased with age (i.e. $6.6 \%$ for those $18-39$ years old, $11.6 \%$ for those $40-49$ years old, $14.0 \%$ for those $50-59$ years old, and $15.9 \%$ for those $>60$ years old) and BMI (i.e. 6.0\% for those with a BMI $<18.5 \mathrm{~kg} / \mathrm{m}^{2}, 9.1 \%$ for those with a BMI between 18.5 and $24.9 \mathrm{~kg} / \mathrm{m}^{2}, 14.7 \%$ for those with a BMI between 25 and $29.9 \mathrm{~kg} / \mathrm{m}^{2}$, and $12.5 \%$ for those with a BMI $\geq 30$ $\mathrm{kg} / \mathrm{m}^{2}$ ). Mongolian-Chinese $(5.1 \%)$ had the lowest prevalence of co-PreHTN and PreDM, whereas both the HanChinese (12.0\%) and Korean-Chinese (11.6\%) demonstrated a two-fold greater risk of co-PreHTN and PreDM than the Mongolian-Chinese. Furthermore, those with a lower educational level, such as primary or secondary education, tended to have a higher prevalence of coPreHTN and PreDM. Co-PreHTN and PreDM participants were more likely to be married, ex-smoking, and current alcohol drinkers $(P<0.0001)$.

\section{Cardiometabolic risk profile}

A summary of the cardiometabolic risk factors of the study population are presented in Table 3, according to their cardiometabolic status (i.e. normotensive and normoglycemic, prehypertensive and prediabetic, or hypertensive and diabetic). SBP $(108.9 \pm 7.2,126.3 \pm 6.9,148.9 \pm 15.6$ $\mathrm{mmHg}$, respectively) and DBP $(70.1 \pm 6.0,80.4 \pm 5.7,91.8$ $\pm 10.1 \mathrm{mmHg}$, respectively; $P<0.0001)$ increased gradually across these categories. Furthermore, there was a steady increase in BMI and WC in both men and women with increasing BP and impaired glucose metabolism. Compared to the normotensive and normoglycemic group, the co-PreHTN and PreDM group had a significantly higher GGT (28.6 [18.0-48.9] U/L), UA (333.4 \pm $86.4 \mu \mathrm{mol} / \mathrm{L})$, and fasting serum glucose $(6.0 \pm 0.3 \mathrm{mmol} /$ $\mathrm{L} ; P<0.0001)$. With respect to the fasting lipid profiles, both TG and TC were significantly lower in normotensive and normoglycemic individuals compared to those with co-PreHTN and PreDM (both $P<0.0001$ ). Additionally, atherogenic LDL-C was significantly lower, whereas antiatherogenic HDL-C was significantly higher (both $P<$ 0.0001 ) in normotensive and normoglycemic individuals versus those with co-PreHTN and PreDM.

Table 4 demonstrates the correlations between BMI, BP, GLU, and lipid profiles with GGT and UA in individuals with co-PreHTN and PreDM. A multivariate correlation analysis demonstrated that GGT and UA levels were significantly and positively associated with BMI, WC, and SBP in the subjects with co-PreHTN and PreDM. Additionally, a significant and positive correlation was observed between serum TG and GGT/UA. Interestingly, a negative correlation was observed between both GGT and UA with HDL-C. A significant positive correlation was also observed between TC and GGT/UA.

\section{Discussion}

In the present study, the population studied presents a high prevalence of co-PreHTN and PreDM in apparently healthy Chinese adults. The prevalence of co-

Table 1 Number of disease-free adults categorized by resting blood pressure and serum glucose levels

\begin{tabular}{|c|c|c|c|c|}
\hline \multirow[b]{2}{*}{ Fasting blood glucose } & \multicolumn{3}{|c|}{ Resting blood pressure } & \multirow[b]{2}{*}{ Total } \\
\hline & $\begin{array}{l}\text { Normal } \\
(<120 / 80 \mathrm{mmHg})\end{array}$ & $\begin{array}{l}\text { Prehypertension } \\
(120-139 / 80-89 \mathrm{mmHg})\end{array}$ & $\begin{array}{l}\text { Hypertension (SBP \&DBP) } \\
(\geq 140 / 90 \mathrm{mmHg})\end{array}$ & \\
\hline Normal $(<5.6 \mathrm{mmol} / \mathrm{L})$ & 2340 & 2625 & 1057 & 6022 \\
\hline Prediabetes (5.6 - $6.9 \mathrm{mmol} / \mathrm{L})$ & 375 & 897 & 498 & 1770 \\
\hline Diabetes ( $\geq 7.0 \mathrm{mmol} / \mathrm{L})$ & 40 & 196 & 160 & 396 \\
\hline Total & 2755 & 3718 & 1715 & 8188 \\
\hline
\end{tabular}

Sample population was classified by blood pressure and glucose status. 
Table 2 Prevalence (\%) of healthy Chinese adults with either normotension and normoglycemia, prehypertension and prediabetes, or hypertension and diabetes mellitus

\begin{tabular}{|c|c|c|c|c|c|c|}
\hline & \multicolumn{2}{|c|}{ Normotension and Normoglycemia } & \multicolumn{2}{|c|}{ PreHTN and PreDM } & \multicolumn{2}{|c|}{ HPT and DM } \\
\hline & $\mathrm{N}$ & $\begin{array}{c}\text { Prevalence } \\
(\%)\end{array}$ & $\mathrm{N}$ & $\begin{array}{c}\text { Prevalence } \\
(\%)\end{array}$ & $\mathbf{N}$ & $\begin{array}{c}\text { Prevalence } \\
(\%)\end{array}$ \\
\hline Overall (2008-2010) & 2340 & $28.6 \pm 0.5$ & 897 & $11.0 \pm 0.3$ & 160 & $2.0 \pm 0.2$ \\
\hline \multicolumn{7}{|l|}{ Survey area } \\
\hline Hei Longjiang province & 1137 & $29.3 \pm 0.6$ & 519 & $13.3 \pm 0.5$ & 97 & $2.5 \pm 0.3$ \\
\hline Inner Mongolian Autonomous Region & 1203 & $27.9 \pm 0.7$ & 378 & $8.8 \pm 0.4$ & 63 & $1.5 \pm 0.2$ \\
\hline \multicolumn{7}{|l|}{ Gender } \\
\hline Men & 645 & $17.9 \pm 0.6$ & 512 & $14.2 \pm 0.6$ & 98 & $2.7 \pm 0.3$ \\
\hline Women & 1695 & $36.9 \pm 0.7$ & 385 & $8.4 \pm 0.4$ & 62 & $1.3 \pm 0.2$ \\
\hline \multicolumn{7}{|l|}{ Age group (years) } \\
\hline $18-39$ & 1438 & $44.7 \pm 0.8$ & 212 & $6.6 \pm 0.4$ & 7 & $0.2 \pm 0.1$ \\
\hline $40-49$ & 495 & $28.1 \pm 1.1$ & 205 & $11.6 \pm 0.8$ & 30 & $1.7 \pm 0.3$ \\
\hline $50-59$ & 268 & $17.0 \pm 0.9$ & 221 & $14.0 \pm 0.9$ & 42 & $2.7 \pm 0.4$ \\
\hline $60-$ & 139 & $8.5 \pm 0.7$ & 259 & $15.9 \pm 0.9$ & 81 & $5.0 \pm 0.5$ \\
\hline \multicolumn{7}{|l|}{ Ethnic group } \\
\hline Han & 1662 & $28.7 \pm 0.6$ & 695 & $12.0 \pm 0.4$ & 112 & $1.9 \pm 0.2$ \\
\hline Korean & 195 & $20.8 \pm 1.3$ & 109 & $11.6 \pm 1.0$ & 34 & $3.6 \pm 0.6$ \\
\hline Mongolian & 395 & $31.9 \pm 1.3$ & 63 & $5.1 \pm 0.6$ & 10 & $0.8 \pm 0.3$ \\
\hline Other & 88 & $38.8 \pm 3.2$ & 30 & $13.2 \pm 2.2$ & 4 & $1.7 \pm 0.8$ \\
\hline \multicolumn{7}{|l|}{ Education } \\
\hline Primary or below & 119 & $14.5 \pm 1.2$ & 100 & $12.2 \pm 1.1$ & 69 & $8.4 \pm 1.0$ \\
\hline Secondary & 874 & $24.3 \pm 0.7$ & 461 & $12.8 \pm 0.6$ & 48 & $1.3 \pm 0.2$ \\
\hline Matriculation or above & 1307 & $36.1 \pm 0.8$ & 320 & $8.8 \pm 0.5$ & 42 & $1.2 \pm 0.2$ \\
\hline \multicolumn{7}{|l|}{ Marital status } \\
\hline Married & 1471 & $25.1 \pm 0.6$ & 724 & $12.4 \pm 0.4$ & 132 & $2.3 \pm 0.2$ \\
\hline Single & 677 & $50.9 \pm 1.3$ & 51 & $3.8 \pm 0.5$ & 3 & $0.2 \pm 0.1$ \\
\hline Divorced/widowed & 85 & $19.3 \pm 1.9$ & 51 & $11.6 \pm 1.5$ & 14 & $3.2 \pm 0.8$ \\
\hline \multicolumn{7}{|l|}{ Smoking } \\
\hline Non-smoker & 1886 & $32.2 \pm 0.6$ & 561 & $9.6 \pm 0.4$ & 110 & $1.9 \pm 0.2$ \\
\hline Current smoker & 357 & $20.1 \pm 0.9$ & 242 & $13.6 \pm 0.8$ & 35 & $2.0 \pm 0.3$ \\
\hline Ex-smoker & 49 & $15.0 \pm 2.0$ & 55 & $16.8 \pm 2.1$ & 13 & $4.0 \pm 1.1$ \\
\hline \multicolumn{7}{|l|}{ Alcohol consumption } \\
\hline Non-drinker & 1825 & $32.6 \pm 0.6$ & 540 & $9.6 \pm 0.4$ & 93 & $1.7 \pm 0.2$ \\
\hline Current drinker & 413 & $19.4 \pm 0.9$ & 290 & $13.6 \pm 0.7$ & 57 & $2.7 \pm 0.3$ \\
\hline Ex-drinker & 38 & $19.5 \pm 2.8$ & 24 & $12.3 \pm 2.3$ & 8 & $4.1 \pm 1.4$ \\
\hline
\end{tabular}

${ }^{1}$ Percent prevalence \pm standard error.

${ }^{2}$ Abbreviations: PreHTN, prehypertension; PreDM, prediabetes; HPT, hypertension; DM, diabetes mellitus.

PreHTN and PreDM was even higher in men than women, and increased with age and BMI. Our findings suggest that prevalence of co-PreHTN and PreDM in our population is similar to that observed in a recent survey conducted in a healthy US population [15]. It has been previously reported that PreDM markedly increases CVD risk in prehypertensive individuals [16]. The recent findings of the Strong Heart Study also revealed that in non-diabetic individuals, IFG and PreHTN increases the number of cardiovascular events by 2.06-fold compared to their normotensive and normoglycemic counterparts, with an absolute increase of 5 cardiovascular events per 1000 person years [10].
It has also been previously shown that there are marked ethnic and geographic differences in BP and FBG levels in China $[9,17]$. Living conditions, habitual diets, and social circumstances in disparate communities also exhibit different characteristics. In the present study, the prevalence of co-PreHTN and PreDM was higher in the Hei Longjiang province of northern China compared to the Inner Mongolian Autonomous Region in northeastern China (13.3\% versus $8.8 \%$ ). There are a number of potential factors that may contribute to these differences. For example, there are more people in the Hei Longiiang province that are overweight or obese, dyslipidemic, and have a higher salt intake [18]. Unfortunately, data on 
Table 3 A comparison of select age-adjusted cardiovascular risk factors in individuals with normotension and normoglycemia, prehypertension and prediabetes, and hypertension and diabetes

\begin{tabular}{|c|c|c|c|c|c|c|c|c|}
\hline & \multicolumn{2}{|c|}{ Normotension-normoglycemia } & \multicolumn{2}{|c|}{ Prehypertension-prediabetes } & \multicolumn{2}{|c|}{ Hypertension-diabetes } & \multirow[b]{2}{*}{$P$-value ${ }^{a}$} & \multirow[b]{2}{*}{$P$-value ${ }^{b}$} \\
\hline & $\mathrm{N}$ & $\bar{x} \pm s / M\left(Q_{\mathrm{R}}\right)$ & $\mathrm{N}$ & $\bar{x} \pm s / M\left(Q_{R}\right)$ & $\mathrm{N}$ & $\bar{x} \pm s / M\left(Q_{R}\right)$ & & \\
\hline \multicolumn{9}{|l|}{ BMI $\left(\mathrm{kg} / \mathrm{m}^{2}\right)$} \\
\hline Men & 645 & $22.5 \pm 3.2$ & 512 & $25.4 \pm 3.5$ & 96 & $26.6 \pm 3.1$ & $<0.0001$ & 0.0018 \\
\hline Women & 1692 & $22.2 \pm 3.9$ & 384 & $24.6 \pm 3.2$ & 61 & $26.8 \pm 3.7$ & $<0.0001$ & $<0.0001$ \\
\hline \multicolumn{9}{|l|}{ WC $(\mathrm{cm})$} \\
\hline Men & 628 & $79.0 \pm 9.7$ & 498 & $88.1 \pm 9.3$ & 97 & $92.2 \pm 8.5$ & $<0.0001$ & $<0.0001$ \\
\hline Women & 1654 & $73.3 \pm 8.3$ & 376 & $82.0 \pm 8.8$ & 60 & $88.7 \pm 9.8$ & $<0.0001$ & $<0.0001$ \\
\hline SBP $(\mathrm{mmHg})$ & 2430 & $108.9 \pm 7.2$ & 897 & $126.3 \pm 6.9$ & 160 & $148.9 \pm 15.6$ & $<0.0001$ & $<0.0001$ \\
\hline $\mathrm{DBP}(\mathrm{mmHg})$ & 2430 & $70.1 \pm 6.0$ & 897 & $80.4 \pm 5.7$ & 160 & $91.8 \pm 10.1$ & $<0.0001$ & $<0.0001$ \\
\hline GGT (U/L) & 2339 & $15.4(12.0-22.9)$ & 897 & 28.6(18.0-48.9) & 160 & $38.1(25.0-64.0)$ & $<0.0001$ & $<0.0001$ \\
\hline UA ( $\mu \mathrm{mol} / \mathrm{L})$ & 2339 & $274.9 \pm 74.6$ & 897 & $333.4 \pm 86.4$ & 160 & $321.3 \pm 78.3$ & $<0.0001$ & 0.0984 \\
\hline GLU (mmol/L) & 2340 & $5.0 \pm 0.4$ & 897 & $6.0 \pm 0.3$ & 160 & $9.1 \pm 2.5$ & $<0.0001$ & $<0.0001$ \\
\hline $\mathrm{TG}(\mathrm{mmol} / \mathrm{L})$ & 2340 & $0.97(0.73-1.35)$ & 897 & $1.58(1.08-2.35)$ & 160 & $2.22(1.52-3.25)$ & $<0.0001$ & $<0.0001$ \\
\hline TC (mmol/L) & 2340 & $4.4 \pm 0.9$ & 897 & $5.0 \pm 1.1$ & 160 & $5.3 \pm 1.1$ & $<0.0001$ & 0.0015 \\
\hline $\mathrm{HDL}-\mathrm{C}(\mathrm{mmol} / \mathrm{L})$ & 2334 & $1.5 \pm 0.3$ & 895 & $1.4 \pm 0.4$ & 160 & $1.3 \pm 0.3$ & $<0.0001$ & 0.0026 \\
\hline LDL-C (mmol/L) & 2340 & $2.3 \pm 0.8$ & 897 & $2.8 \pm 0.9$ & 160 & $3.1 \pm 0.9$ & $<0.0001$ & 0.0001 \\
\hline
\end{tabular}

${ }^{1} \mathrm{GGT}$ and TG were reported as medians (interquartile range).

${ }^{2}$ Age-adjusted by analysis of covariance.

${ }^{3}$ Abbreviations: BMI, body mass index; WC, waist circumference; SBP, systolic blood pressure; DBP, diastolic blood pressure; GGT, $\gamma$ - glutamyltransferase; UA, uric acid; GLU, glucose; TG, triglyceride; TC, total cholesterol; HDL-C, high density lipoprotein cholesterol; LDL-C, low density lipoprotein cholesterol.

${ }^{\text {a }}$ Normotension-normoglycemia vs. prehypertension-prediabetes

b Prehypertension-prediabetes vs. hypertension-diabetes

dietary sodium intake were not collected in the present study. Furthermore, based on the regional differences, there may be genetic contributions to the co-PreHTN and PreDM phenotype that should also be considered.

There was a higher prevalence of co-PreHTN and PreDM among individuals that consumed alcohol and smoked. This finding was consistent with earlier studies, which found that excessive alcohol consumption and

Table 4 Correlations between various relevant factors and serum GGT and UA in prehypertensive and prediabetic individuals

\begin{tabular}{lllll}
\hline Parameters & \multicolumn{3}{c}{ GGT } & \multicolumn{2}{c}{ UA } \\
\cline { 2 - 5 } & $\boldsymbol{r}$ & $\boldsymbol{p}$ & $\boldsymbol{r}$ & $\boldsymbol{p}$ \\
\hline BMI & 0.375 & 0.000 & 0.289 & 0.000 \\
WC & 0.434 & 0.000 & 0.405 & 0.000 \\
SBP & 0.041 & 0.215 & 0.074 & 0.026 \\
DBP & 0.148 & 0.000 & 0.071 & 0.333 \\
GLU & 0.169 & 0.000 & 0.041 & 0.215 \\
TG & 0.454 & 0.000 & 0.316 & 0.000 \\
TC & 0.251 & 0.000 & 0.139 & 0.000 \\
HDL-C & -0.206 & 0.000 & -0.236 & 0.000 \\
LDL-C & 0.167 & 0.000 & 0.047 & 0.157 \\
\hline
\end{tabular}

Abbreviations: GGT, $\gamma$ - glutamyltransferase; UA, uric acid; BMI, body mass index; WC, waist circumference; SBP, systolic blood pressure; DBP, diastolic blood pressure; GLU, glucose; TG, triglyceride; TC, total cholesterol; HDL-C, high density lipoprotein cholesterol; LDL-C, low density lipoprotein cholesterol. cigarette smoking increase BP and glucose levels $[19,20]$. Interestingly, we also found that there was higher prevalence of ex-smokers than current smokers in the coPreHTN and PreDM group. This observation can be explained by the findings of Inoue K [21], who suggested that the adverse effects associated with the weight gain following cessation of smoking outweigh the benefits. Weight gain is a strong risk factor for CVD, which is also closely associated with a substantial risk for impaired glucose regulation and elevated BP [22].

In the present study, we also observed that, with the exception of HDL-C, the lipid profile and glucose levels demonstrated a gradual upward trend from the normotensive and normoglycemic group, followed by the coPreHTN and PreDM group, and lastly the hypertensive and diabetic group. In addition to the larger WC and BMI, our findings also suggest that these considerably "healthy" men and women are at an increased risk for CVD. Other biochemical parameters, such as GGT and UA levels, were also relatively higher in subjects with co-PreHTN and PreDM. Recent cross-sectional and longitudinal studies have also noted a relatively independent association between elevated serum GGT levels, and hypertension and diabetes $[23,24]$. We have also revealed that higher serum GGT levels are positively associated with PreHTN in an otherwise healthy Chinese adult population following various adjustments in the multivariate logistic regression analysis (unpublished observations). In addition, a recent 
study [25] found that elevated GGT was independently associated with the presence of the IFG, further supporting our conclusion. Similarly, serum UA levels correlated with metabolic abnormalities and CVD [26]. A previous study [27] found that there is a significant association between serum UA levels and the risk of PreHTN. Meanwhile, in the Rancho Bernardo Study, it was suggested that UA may be a useful predictor of type 2 diabetes in older adults with IFG [28]. However, the underlying mechanisms for higher GGT and UA in subjects with co-PreHTN and PreDM are unclear and require further clarification.

A major strength of our study is that it is a population-based study with a good representative sample of the general Chinese population. Additionally, the larger sample size ensures sufficient power in estimating the prevalence of co-PreHTN and PreDM, as well as determining the correlation between co-PreHTN and PreDM, and CVD risk factors. Lastly, the use of standardized protocols and instruments guaranteed a high response rate and excellent quality control.

However, the study also has several limitations. First, oral glucose tolerance test (OGTT) was not performed in subjects with IFG, thereby potentially reducing the accurate diagnosis of diabetes. Since some individuals with normal FBG may have IGT, based on OGTT data, examining FBG alone (i.e. without a 2-h PBG) may result in the underestimating of the prevalence of co-PreHTN and PreDM in healthy Chinese adults. Lastly, the study is based on a cross-sectional survey, which is unable to determine causality or the temporal relationship between CVD risk factors and co-PreHTN and PreDM.

\section{Conclusions}

Our data highlights the high prevalence of co-PreHTN and PreDM in disease-free Chinese adults from northern and northeastern China. With the exception of HDL-C, higher lipid profile levels, as well as GGT, UA, and FBG, in coPreHTN and PreDM individuals may have important pathophysiological implications. All of these changes have been shown to increase CVD risk, and thereby suggests that there is an urgent need for early detection and appropriate interventions. A healthy lifestyle, such as weight control, increased physical activity, moderate alcohol intake, tobacco cessation, salt reduction, and sufficient consumption of fresh fruits and vegetables could prevent the progression of co-PreHTN and PreDM to overt hypertension and diabetes mellitus. Public health programs are required to improve this situation in the Chinese population.

\section{Acknowledgements}

The present study was supported by the Special Fund for Key Basic Research Project of the Ministry of Science and Technology of China (2006FY110300). We are also grateful to the participants, primary care doctors and nurses who took part in the survey.

\section{Author details}

'Department of Clinical Laboratory, Peking Union Medical College Hospital, Peking Union Medical College \& Chinese Academy of Medical Science, Beijing 100730, China. ${ }^{2}$ Department of Endocrinology, Chinese PLA General Hospital, Beijing 100853, China. ${ }^{3}$ Department of Clinical Laboratory, The $2^{\text {nd }}$ Affiliated Hospital of Harbin Medical University, Harbin 150001, China. ${ }^{4}$ Department of Clinical Laboratory, Inner Mongolian People's Hospital, Hohhot, 010017, China. Institute of Basic Medical Sciences, Chinese Academy of Medical Sciences \& Peking Union Medical College, Beijing 100005, China

\section{Authors' contributions}

JW carried out the experimental design, analysis and interpretation of data, and drafted the manuscript. WHY carried out the experimental design, performed statistical analysis, and drafted the manuscript. LQ conceived the study, participated in its design and coordination, and helped in drafting the manuscript. XQC, XZG, WW, LYX, and XZQ participated in the study and manuscript revision. YHL and HTD were responsible for the acquisition of data and manuscript revision. SMH, CLX, and GJZ participated in the design of the study and performed the statistical analysis. All authors read and approved the final manuscript.

\section{Competing interests}

The authors declare that they have no competing interests.

Received: 12 August 2011 Accepted: 12 October 2011

Published: 12 October 2011

\section{References}

1. He J, Gu D, Wu X, Reynolds K, Duan X, Yao C: Major causes of death among men and women in China. N Engl J Med 2005, 353:1124-1134.

2. Lewington S, Clarke R, Qizilbash N, Peto R, Collins R: Age-specific relevance of usual blood pressure to vascular mortality: a meta-analysis of individual data for one million adults in 61 prospective studies. Lancet 2002, 360:1903-1913.

3. Chobanian AV, Bakris GL, Black HR, Cushman WC, Green LA: The Seventh Report of the Joint National Committee on Prevention, Detection, Evaluation, and Treatment of High Blood Pressure: the JNC 7 report. JAMA 2003, 289:2560-2572.

4. Grotto I, Grossman E, Huerta M, Sharabi Y: Prevalence of prehypertension and associated cardiovascular risk profiles among young Israeli adults. Hypertension 2006, 48:254-259.

5. Wang $Y$, Wang QJ: The prevalence of prehypertension and hypertension among US adults according to the new joint national committee guidelines: new challenges of the old problem. Arch Intern Med 2004, 164:2126-2134.

6. Choi KM, Park HS, Han JH, Lee JS, Lee J, Ryu OH: Prevalence of prehypertension and hypertension in a Korean population: Korean National Health and Nutrition Survey 2001. J Hypertens 2006, 24:1515-1521.

7. American Diabetes Association: Diagnosis and classification of diabetes mellitus. Diabetes Care 2011, 34(Suppl 1):S62-69.

8. Nichols GA, Hillier TA, Brown JB: Progression from newly acquired impaired fasting glusose to type 2 diabetes. Diabetes Care 2007, 30:228-233.

9. Yang W, Lu J, Weng J, Jia W, Ji L, Xiao J: Prevalence of diabetes among men and women in China. N Engl J Med 2010, 362:1090-1101.

10. Zhang Y, Lee ET, Devereux RB, Yeh J, Best LG, Fabsitz RR: Prehypertension, diabetes, and cardiovascular disease risk in a population-based sample: the Strong Heart Study. Hypertension 2006, 47:410-414.

11. Okosun IS, Boltri JM, Anochie LK, Chandra KM: Racial/ethnic differences in prehypertension in American adults: population and relative attributable risks of abdominal obesity. J Hum Hypertens 2004, 18:849-855.

12. Player MS, Diaz VA, Mainous lii AG, Gregorie SH, Knoll ME, Everett CJ: Ethnic differences in the relationship of prediabetes with the presence of target-organ disease. Diabetes Metab 2011.

13. Yang J, Lu F, Zhang C, Liu Z, Zhao Y, Gao F: Prevalence of prehypertension and hypertension in a Chinese rural area from 1991 to 2007. Hypertens Res 2010, 33:331-337.

14. Physical status: the use and interpretation of anthropometry. Report of a WHO Expert Committee.: World Health Organ Tech Rep Ser 1995, 854:1-452. 
15. Gupta AK, Brashear MM, Johnson WD: Coexisting prehypertension and prediabetes in healthy adults: a pathway for accelerated cardiovascular events. Hypertens Res 2011, 34:456-461.

16. Barr EL, Zimmet PZ, Welborn TA, Jolley D, Magliano DJ, Dunstan DW: Risk of cardiovascular and all-cause mortality in individuals with diabetes mellitus, impaired fasting glucose, and impaired glucose tolerance: the Australian Diabetes, Obesity, and Lifestyle Study (AusDiab). Circulation 2007, 116:151-157.

17. Meng XJ, Dong GH, Wang D, Liu MM, Lin Q, Tian S: Prevalence, awareness, treatment, control, and risk factors associated with hypertension in urban adults from 33 communities of China: the CHPSNE study. J Hypertens 2011, 29:1303-1310.

18. Zhao L, Stamler J, Yan LL, Zhou B, Wu Y, Liu K: Blood pressure differences between northern and southern Chinese: role of dietary factors: the International Study on Macronutrients and Blood Pressure. Hypertension 2004, 43:1332-1337.

19. Matsui Y, Kario K, Ishikawa J, Hoshide S, Eguchi K, Shimada K: Smoking and antihypertensive medication: interaction between blood pressure reduction and arterial stiffness. Hypertens Res 2005, 28:631-638.

20. Sargeant LA, Khaw KT, Bingham S, Day NE, Luben RN, Oakes S: Cigarette smoking and glycaemia: the EPIC-Norfolk Study. Int J Epidemiol 2001, 30:547-554, European Prospective Investigation into Cancer.

21. Inoue K, Takeshima F, Kadota K, Yoda A, Tatsuta Y, Nagaura Y: Early effects of smoking cessation and weight gain on plasma adiponectin levels and insulin resistance. Intern Med 2011, 50:707-712.

22. Yoon C, Goh E, Park SM, Cho B: Effects of smoking cessation and weight gain on cardiovascular disease risk factors in Asian male population. Atherosclerosis 2010, 208:275-279.

23. Lee DH, Jacobs DR Jr, Gross M, Kiefe Cl, Roseman J, Lewis CE: Gammaglutamyltransferase is a predictor of incident diabetes and hypertension: the Coronary Artery Risk Development in Young Adults (CARDIA) Study. Clin Chem 2003, 49:1358-1366.

24. Onat A, Can G, Ornek E, Cicek G, Ayhan E, Dogan Y: Serum gammaGlutamyltransferase: Independent Predictor of Risk of Diabetes, Hypertension, Metabolic Syndrome, and Coronary Disease. Obesity (Silver Spring); 2011.

25. Sun J, Ren J, Pang ZC, Gao WG, Nan HR, Wang SJ: The association of gamma-glutamyltransferase and C-reactive protein with IFG/IGT in Chinese adults in Qingdao, China. Clin Chim Acta 2011, 412:1658-1661.

26. Feig DI, Kang DH, Johnson RJ: Uric acid and cardiovascular risk. N Engl J Med 2008, 359:1811-1821.

27. Syamala S, Li J, Shankar A: Association between serum uric acid and prehypertension among US adults. J Hypertens 2007, 25:1583-1589.

28. Kramer CK, von Muhlen D, Jassal SK, Barrett-Connor E: Serum uric acid levels improve prediction of incident type 2 diabetes in individuals with impaired fasting glucose: the Rancho Bernardo Study. Diabetes Care 2009, 32:1272-1273.

\section{Pre-publication history}

The pre-publication history for this paper can be accessed here: http://www.biomedcentral.com/1471-2458/11/794/prepub

\section{Submit your next manuscript to BioMed Central and take full advantage of:}

- Convenient online submission

- Thorough peer review

- No space constraints or color figure charges

- Immediate publication on acceptance

- Inclusion in PubMed, CAS, Scopus and Google Scholar

- Research which is freely available for redistribution

Submit your manuscript at www.biomedcentral.com/submit 